\title{
Ueber drei malayische Trematoden
}

\author{
(SUR trois Trématodes de Malaisie)
}

\author{
Par Klaus ROHDE, Siew Kein LEE et Heng Wan LIM \\ (Institut für Allgemeine Zoologie Ruhr-Universität, 463 Bochum, Allemagne)
}

\begin{abstract}
Résumé
Les Trématodes de Malaisie qui suivent sont décrits: Zonorchis sp. de Callosciurus notatus et $C$. caniceps, Coeuritrema macrotesticularis n. sp. de Dogania subplana et Spirhapalum elongatum n. sp. de Cyclemys amboinensis.
\end{abstract}

\section{Summary}

The following Malayan trematodes are described: Zonorchis sp. from Callosciurus notatus and $C$. caniceps, Coeuritrema macrotesticularis n. sp. from Dogania subplana, Spirhapalum elongatum n. sp. from Cyclemys amboinensis.

Die im folgenden beschriebenen Würmer wurden in den Jahren 1961-1966 aus verschiedenen malayischen Tieren gesammelt, in Bouins Fixierungsflüssigkeit fixiert und mit Alaun-Karmin nach Grenacher gefärbt. Einzelheiten der Geschlechtsorgane wurden aus mit Azan gefärbten Serienschnitten rekonstruiert. 


\section{ZONORCHIS sp.}

Beschreibung. Flach, grösste Breite auf der Höhe der Hoden oder dicht dahinter, nach vorne und hinten zu schmaler werdend. Mundsaugnapf subterminal, Pharynx und Oesophagus vorhanden. Larmblindsäcke nicht ganz bis zum Körperhinterende, manchmal von verschiedener Länge. Acetabulum im ersten Köperdrittel, sehr gross. Cirrussack zwischen Acetabulum und Pharynx, mit ausstülpbarem Cirrus. Geschlechtsöffnung am Hinterrande des Pharynx oder dicht dahinter. Hoden ganzrandig, sich unmittelbar hinter dem Acetabulum gegenüber liegend. Ovar schräg hinter dem einen Hoden, gelappt, entweder den Hoden berührend oder von ihm durch Uterusschlingen getrennt. Receptaculum seminis und Mehlissche Drüse hinter dem Ovar. Uterus füllt den Raum zwischen den Darmblindsäcken vom Acetabulum bis zum Körperhinterende aus. Dotterstöcke lateral von den Darmblindsäcken, meist vor der Ebene der Hoden, in einigen Fällen auf der Höhe des Vorderrandes der Hoden beginnend, bis dicht vor das Ende oder bis zum Ende der Darmblindsäcke. Eier oval, mit Operculum. (Abb. 1).

Messergebnisse. (In Millimetern, an 5 Exemplaren, Längs - vor dem Querdurchmesser) :

\begin{tabular}{|c|c|c|c|c|c|c|}
\hline Länge & 2.00 & -3.84 & (Durchschnitt & 3.07) & & \\
\hline Max. Breite & 1.00 & -1.20 & (Durchschnitt & 1.11) & & \\
\hline Mundsaugnapf $\ldots \ldots \ldots \ldots$ & 0.18 & -0.21 & $\times 0.18-0.25$ & (Durchschnitt & 0.20 & $\times 0.22$ \\
\hline Acetabulum & 0.34 & -0.44 & $\times 0.40-0.48$ & (Durchschnitt & 0.41 & $\times 0.44$ \\
\hline Cirrussack & 0.20 & -0.28 & $\times 0.07-0.11$ & (Durchschnitt & 0.25 & 0.09 \\
\hline Hoden (ovarial) & 0.36 & -0.55 & $\times 0.28-0.41$ & (Durchschnitt & 0.43 & 0.35 \\
\hline Hoden ... & 0.32 & -0.50 & $\times 0.33-0.38$ & (Durchschnitt & 0.41 & $\times 0.34$ \\
\hline Ovar $\ldots$. & 0.12 & -0.22 & $\times 0.19-0.23$ & (Durchschnitt & 0.17 & 0.22 \\
\hline Rec. sem. $\ldots \ldots \ldots \ldots \ldots$ & 0.10 & $\times 0$. & $99-0.10$ & (Durchschnitt & 0.10 & 0.10 \\
\hline Pharynx $\ldots \ldots \ldots \ldots \ldots$ & 0.10 & -0.13 & $\times 0.11-0.12$ & (Durchschnitt & 0.12 & 0.12 \\
\hline Eier .. & 0.02 & -0.031 & $\times 0.017-0.018$ & (Durchschnitt & 0.028 & $\times 0.01$ \\
\hline
\end{tabular}

Wirt : Callosciurus notatus (Boddaert) und C. caniceps (Gray).

Organ: Gallengänge, gelegentlich Darm.

Fundorte: Bukit Lagong, Subang, Ulu Langat und andere Lokalitäten in Malaya.

Exemplare in Helminthological Collection, Zoology Department, University of Malaya, Kuala Lumpur.

Verwandtschaft. Eine Abgrenzung von den anderen bekannten Formen lässt sich zur Zeit wegen der zahlreichen, zum Teil nur unvollständig beschriebenen Arten nicht vornehmen (vgl. z. B. skrjabin 1952, Jaiswal 1957, und Breues und Jimenez Quiros 1959). 


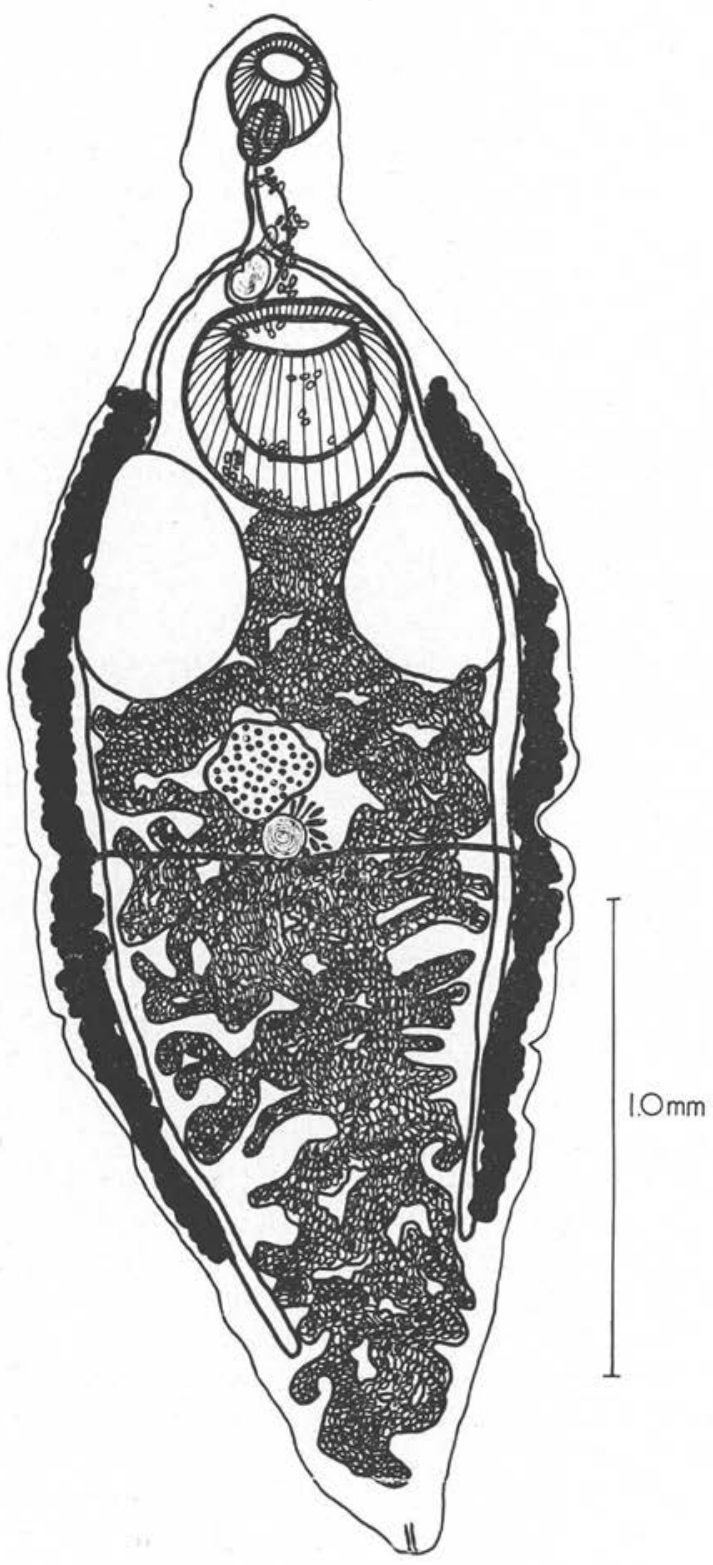

Авв. 1: Zonorchis sp. 


\section{COEURITREMA MACROTESTICULARIS n. sp.}

Beschreibung. (Auf 10 Exemplaren beruhend). Lang und flach, maximale Breite etwa am Äquator, nach vorne und hinten zu schmaler werdend. Mundsaugnapf terminal oder subterminal, kleiner als das Acetabulum. Acetabulum im ersten Viertel oder Drittel des Körpers. Cirrussack gross, schräg bis quer, hinter dem Acetabulum. Kein Pharynx, doch wulstartige Anhäufung von kleinen Zellkernen am Hinterrande des Mundsaugnapfes. Oesophagus lang, von drüsenartigen Zellen umgeben, die zwei deutliche Anschwellungen bilden. Darmverzweigung vor dem Acetabulum. Darmschenkel wellenförmig, nahe dem hinteren Körperende endigend, ein Darmschenkel auf der Hôhe des Cirrusackes deutlich nach innen umbiegend. Exkretionsblase median am Hinterende des Körpers, mit seitlichen Ausbuchtungen, sich am Hinterende der Darmschenkel verzweigend. Dotterstöcke aus grossen Follikeln bestehend, hauptsächlich lateral von den Darmschenkeln, vor dem Acetabulum und hinter dem hinteren Hoden in der Mittellinie zuzammenstossend. Zwei Hoden, stark gelappt, intercaecal, hintereinander, in den mittleren zwei Fünfteln des Körpers, durch das Ovar voneinander getrennt. Querverlaufende Dottergänge hinter dem Ovar. Receptaculum seminis vor dem hinteren Hoden. Geschlechtsöffnung dorsolateral. (Abb. 2-4).

Wirt : Dogania subplana (Geoffr.).

Organ: Herz oder Arterien nahe dem Herzen.

Fundort: Malaya. (Die Schildkröten wurden in einem chinesischen Laden in Kuala Lumpur, Malaya, gekauft).

Holotyp : Helminthological Collection No. R. 769. Zoology Dept., University of Malaya, Kuala Lumpur.

Verwandtschaft. (Der Diskussion liegt das System Skrjabins 1951 zugrunde. Die neue Art gehört zur Gattung Coeuritrema Mehra, 1933 (Synonym Tremarhynchus Thapar, 1933), Spirorchidae, Hapalotrematinae Stunkard, 1921. Diese Gattung ist charakterisiert durch ein Acetabulum, das grösser als der Mundsaugnapf ist und ein Drittel der Körperlänge hinter dem Vorderende liegt. Der Mundsaugnapf ist ausstülpbar, ein Pharynx fehlt und der Oesophagus ist von Drüzenzellen umgeben und mässig lang. Der Darm verzweigt sich unmittelbar vor dem Acetabulum, er bildet charakteristische Schleifen hinter dem Bauchsaugnapf. Die Geschlechtsöffnung liegt dorsal auf der linken Körperseite hinter dem Acetabulum. Zwei Hoden liegen im dritten Köperviertel, das Ovar liegt zwischen ihnen. Der Cirrussack ist praeacetabular. Die Dotterstöcke liegen lateral und in der Zone der Darmschenkel und stossen in der Mittellinie hinter dem Bauchsaugnapf und hinter dem hinteren Hoden zusammen.

Bisher sind 3 Arten dieser Gattung beschrieben worden, die sich in den folgenden hauptsächliden Merkmalen von der neuen Art unterscheiden (zit. nach Skrjabin 1951). 

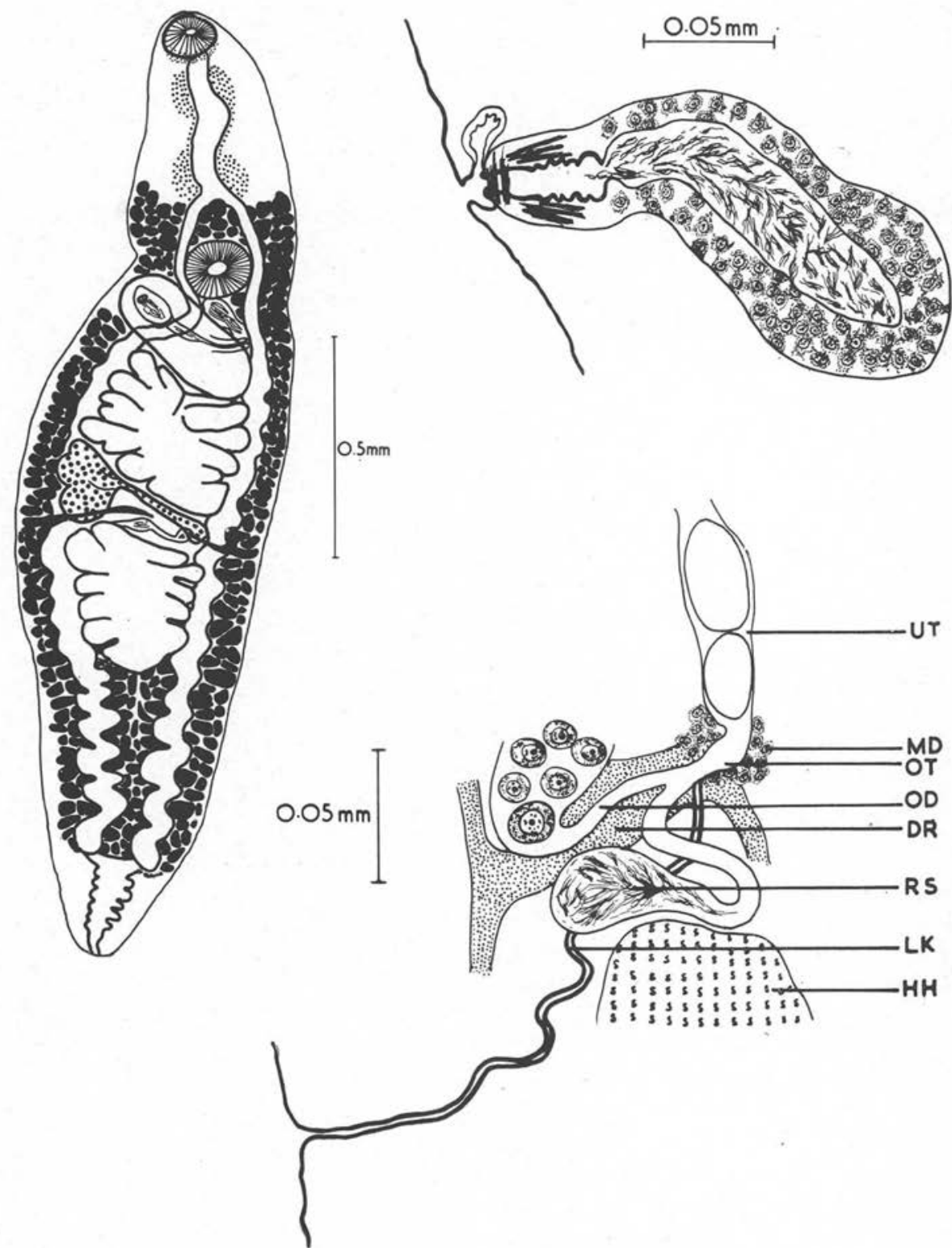

Aвв. 2: Coeuritrema macrotesticularis n. sp.

AвB. 3: Cirrussack von Coeuritrema macrotesticularis.

Aвв. 4: Ootyp und umliegende Geschlechtsgänge von Coeuritrema macrotesticularis. Ventralansicht. UT $=$ Uterus, $\mathrm{MD}=$ Mehlissche Drüse, OT $=$ Ootyp, $\mathrm{OD}=$ Ovidukt, $\mathrm{DR}=$ Dotterreservoir, $\overline{R S}=$ Receptaculum seminis, LK $=$ Laurerscher Kanal, $\mathrm{HH}=$ Hinterer Hoden. 


\begin{tabular}{|c|c|c|c|c|c|c|c|c|c|c|}
\hline$\infty$ & $\underset{i}{i}$ & $\stackrel{0}{0}$ & 1 & $\begin{array}{l}\frac{0}{0} \\
\times \\
\frac{n}{0}\end{array}$ & $\begin{array}{l}\frac{n}{0} \\
\times \\
m \\
0 \\
0\end{array}$ & & $\begin{array}{l}0 \\
\tilde{0} \\
0 \\
\times \\
0 \\
0 \\
0\end{array}$ & & $\begin{array}{l}\text { Jे } \\
\text { bे } \\
\times \\
\dot{\sigma} \\
\dot{0}\end{array}$ & $\begin{array}{l}\frac{n}{0} \\
\times \\
\tilde{I} \\
0\end{array}$ \\
\hline$r$ & $\underset{i}{i}$ & $\frac{0}{0}$ & $\begin{array}{l} \pm \\
\dot{0} \\
x \\
\frac{m}{0}\end{array}$ & $\begin{array}{l}0 \\
0 \\
x \\
0 \\
0\end{array}$ & $\begin{array}{l}\frac{\infty}{0} \\
\dot{0} \\
\times \\
\dot{0} \\
\tilde{b}\end{array}$ & & 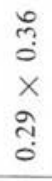 & & $\begin{array}{l}0 \\
0 \\
0 \\
x \\
\tilde{t} \\
0\end{array}$ & 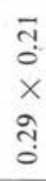 \\
\hline 0 & $\begin{array}{l}\stackrel{0}{n} \\
\text { i }\end{array}$ & है & 1 & $\begin{array}{l}\frac{0}{0} \\
\times \\
\frac{m}{0}\end{array}$ & $\begin{array}{l}\frac{0}{5} \\
\times \\
\hat{n} \\
\vdots\end{array}$ & & $\begin{array}{l}n \\
\tilde{0} \\
\times \\
0 \\
\text { o } \\
0\end{array}$ & & $\begin{array}{l}q \\
q \\
0 \\
\times \\
n \\
q \\
0\end{array}$ & \begin{tabular}{l}
$\frac{N}{0}$ \\
$\dot{x}$ \\
0 \\
\cline { 1 - 1 } \\
0
\end{tabular} \\
\hline$n$ & $\frac{\infty}{i}$ & లి & $\begin{array}{l} \pm \\
0 \\
x \\
0 \\
0\end{array}$ & $\begin{array}{l} \pm \\
0 \\
x \\
\frac{m}{0}\end{array}$ & $\begin{array}{l}8 \\
0 \\
\times \\
\times \\
\grave{0} \\
0\end{array}$ & & 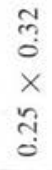 & & 1 & $\begin{array}{l}g \\
\dot{0} \\
x \\
\tilde{y} \\
\tilde{0}\end{array}$ \\
\hline v & $\frac{ \pm}{i}$ & 号 & $\begin{array}{l}\frac{U}{0} \\
x \\
= \\
=\end{array}$ & $\begin{array}{l}0 \\
0 \\
\times \\
\pm \\
0\end{array}$ & $\begin{array}{l}\hat{\jmath} \\
0 \\
x \\
0 \\
\tilde{0} \\
0\end{array}$ & & $\begin{array}{l}0 \\
\text { jo } \\
\times \\
0 \\
0 \\
0\end{array}$ & & $\begin{array}{l}\text { సे } \\
0 \\
\times \\
0 \\
0 \\
0\end{array}$ & $\begin{array}{l}\frac{N}{0} \\
\times \\
\infty \\
0\end{array}$ \\
\hline$m$ & $\overline{\bar{i}}$ & $\stackrel{\circ}{\circ}$ & $\begin{array}{l} \pm \\
0 \\
\times \\
= \\
0\end{array}$ & $\begin{array}{l}I \\
0 \\
\times \\
\pm \\
0\end{array}$ & $\begin{array}{l}\frac{n}{0} \\
\times \\
\times \\
\infty \\
\stackrel{0}{0}\end{array}$ & & $\begin{array}{l}\hat{n} \\
0 \\
x \\
\hat{\jmath} \\
0\end{array}$ & & $\begin{array}{l}0 \\
\text { ? } \\
\times \\
\text { तै } \\
? \\
?\end{array}$ & $\begin{array}{l}= \\
0 \\
\times \\
\frac{a}{0}\end{array}$ \\
\hline$N$ & $\stackrel{\infty}{\circ}$ & $\underset{0}{\overrightarrow{0}}$ & $\begin{array}{l}= \\
0 \\
\times \\
0 \\
0\end{array}$ & $\begin{array}{l}= \\
0 \\
\times \\
0 \\
0\end{array}$ & 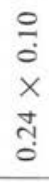 & & $\begin{array}{l}\text { Î } \\
0 \\
x \\
\infty \\
\frac{\infty}{0}\end{array}$ & & 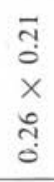 & $\begin{array}{l}5 \\
0 \\
\times \\
x \\
0 \\
0\end{array}$ \\
\hline- & $\hat{o}$ & $\frac{0}{0}$ & $\begin{array}{l}0 \\
0 \\
0 \\
\times \\
0 \\
0 \\
0\end{array}$ & 1 & 1 & & $\begin{array}{l}\stackrel{0}{0} \\
0 \\
\times \\
0 \\
0 \\
0\end{array}$ & & $\begin{array}{l}0 \\
0 \\
x \\
\pm \\
\dot{0}\end{array}$ & 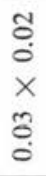 \\
\hline & \begin{tabular}{c}
$\vdots$ \\
$\vdots$ \\
$\vdots$ \\
: \\
: \\
\multirow{J}{*}{}
\end{tabular} & 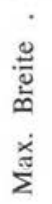 & 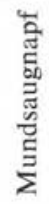 & 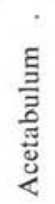 & 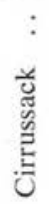 & 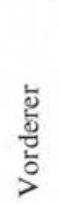 & $\begin{array}{l}: \\
\text { 몽 } \\
\text { 옴 }\end{array}$ & 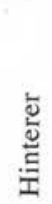 & 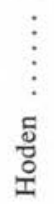 & $\begin{array}{c}\vdots \\
\vdots \\
\vdots \\
\text { ठ }\end{array}$ \\
\hline
\end{tabular}


C. lyssimus Mehra, 1933. Indien. 1.53-1.92 mm lang, Mundsaugnapf 0.1$0.12 \mathrm{~mm}$ Durchmesser, Acetabulum 0.17-0.19 mm Durchmesser, beide Hoden in der hinteren Körperhälfte, vorderer Hoden rechts von der Medianlinie, hinterer Hoden nur schwach gelappt oder rundlich, Cirrussack 0.15-0.24 $\times 0.045-0.084 \mathrm{~mm}$.

C. indicus (Thapar, 1933) Mehra, 1934. 3.16-3.45 mm lang, hinterer Hoden grösser als vorderer. Hoden relativ viel kleiner.

C. odhnerensis Mehra, 1933. Indien. $1.5 \mathrm{~mm}$ lang. Hoden rundlich, deutlich gelappt, in der hinteren Korperhälfte, relativ viel kleiner. Cirrussack $0.18 \times 0.054 \mathrm{~mm}$. Yamaguti (1958) nennt die Gattung Tremarhynchus Thapar, 1933, Unterfamilie Tremarhynchinae Yamaguti, 1958. Er ordnet Hapalorhynchus yoshidai Ozaki, 1939, aus China, neben den drei bereits genannten Arten diesem Genus ein. Hauptsächliche Unterschiede zwischen dieser und der neuen Art sind : die Dotterstöcke beginnen hinter der Darmverzweigung, die Geschlechtsöffnung liegt weiter hinten, die Hoden sind nicht gelappt.

\section{SPIRHAPALUM ELONGATUM n. sp.}

Beschreibung. (Auf 26 Exemplaren beruhend).

Flach und lang, grösste Breite hinter dem Äquator, nach vorne und hinten zu schmaler werdend. Mundsaugnapf rudimentär. Oesophagus quergestreift, lang, wellenförming, von drüsenartigen Zellen umgeben. Acetabulum am Anfang des zweiten Körperdrittels. Darmblindsäcke endigen nahe dem Hinterende. 6 Hoden praeovarial, 1 Hoden postovarial, stark gelappt, intercaecal, median. Ovar submedian, stark gelappt, im hinteren Körperviertel. Uterus kurz, mit 1 Ei. Vordere Samenblase dreigeteilt, latero-frontal vor dem Ovar. Die beiden hinteren Teile in Cirrusbeutel, der auch ausstülpharen Cirrus enthält. Hintere Samenblase hinter dem Ovar. Dotterstöcke von der Darmverzweigung bis zum hinteren Körperende, hauptsächlich lateral von den Darmschenkeln und in der Zone der Darmschenkel, vor den vorderen Hoden auch intercaecal, nur einen schmalen Raum in der Mittellinie freilassend, hinter der Darmverzweigung und am Hinterende des Körpers in der Mittellinie zusammenstossend, einige grosse Follikel intercaecal auf der Höhe des Ovars und vor dem hinteren Hoden. Geschlechtsöffnung ventrolateral. (Abb. 5-8).

Wirt : Cyclemys amboinensis (Daud.)

Organ: Arterien des Mesenteriums.

Fundort: Tanjong Karang, Malaya (Typlokalität); Jesselton, Nordbornèo.

Syntypen: Helminthological Collection No. R. 779-785. Zoology Department, University of Malaya, Kuala Lumpur.

Verwandtschaft. Die Gattung Spirhapalum, Ejsmont, 1925, Spirorchinae, Stunkard, 1921, zu der die neue Art gehört, hat die folgenden hauptsächlichen Kennzeichen : kein Pharynx, Oesophagus von drüsenartigen Zellen umgeben, Darmschenkel bis zum Körperhinterende, Hoden in 2 Gruppen, einer vorderen und einer hinteren, voneinander 
durch das Ovar und die Gesch'echtsgänge getrennt. Vordere Gruppe der Hoden besteht aus kleinen Teilhoden (6), die hintere Gruppe aus 1-2 Teilen. Mit mächtig entwickeltem Cirrussack, männliche Geschlechtsöffnung ventral nahe dem linken Darmschenkel ; gelapptes Ovar hinter der vorderen Gruppe von Hoden auf der rechten

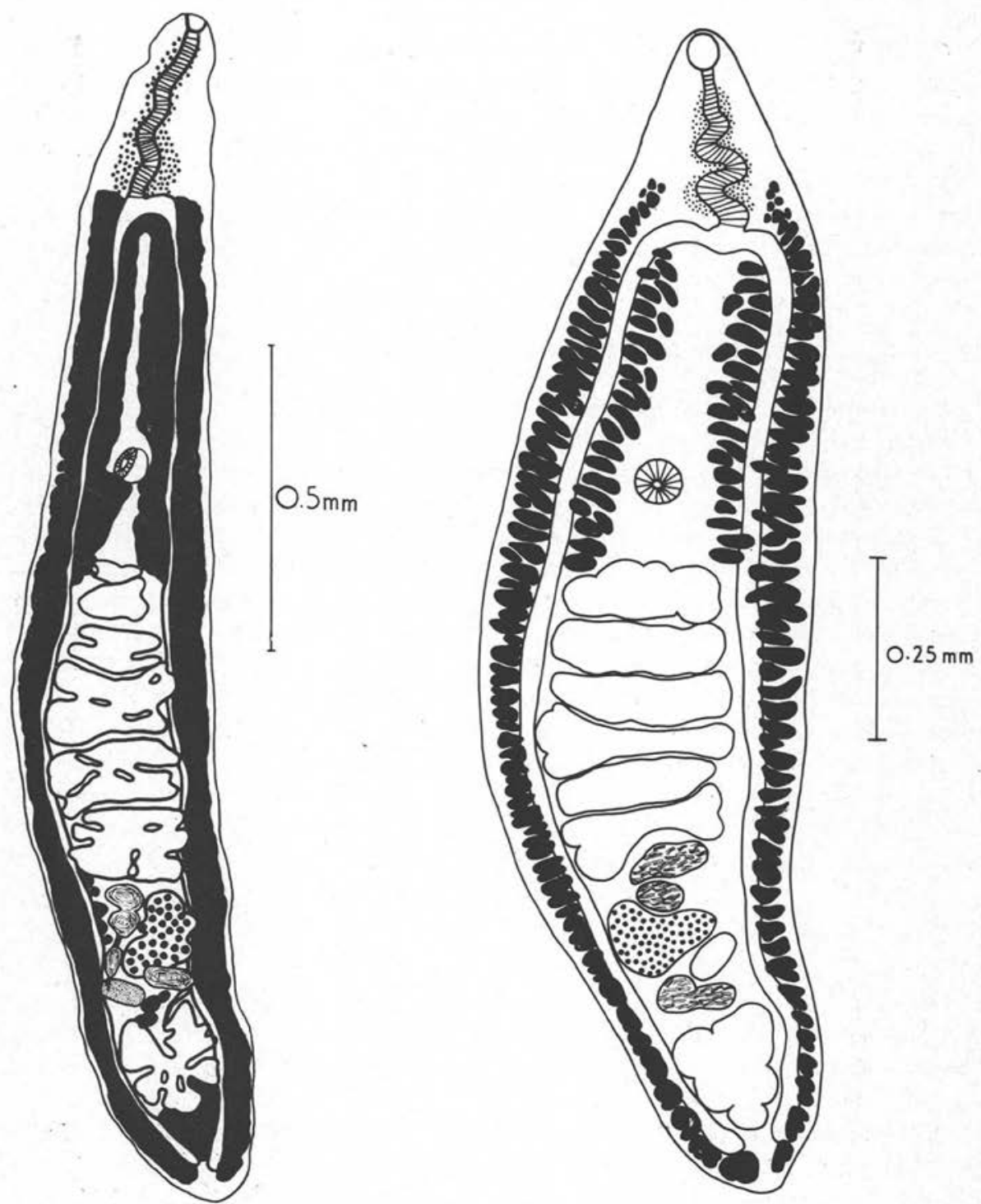

AвB. 5 und 6: Spirhapalum elongatum n. sp. 


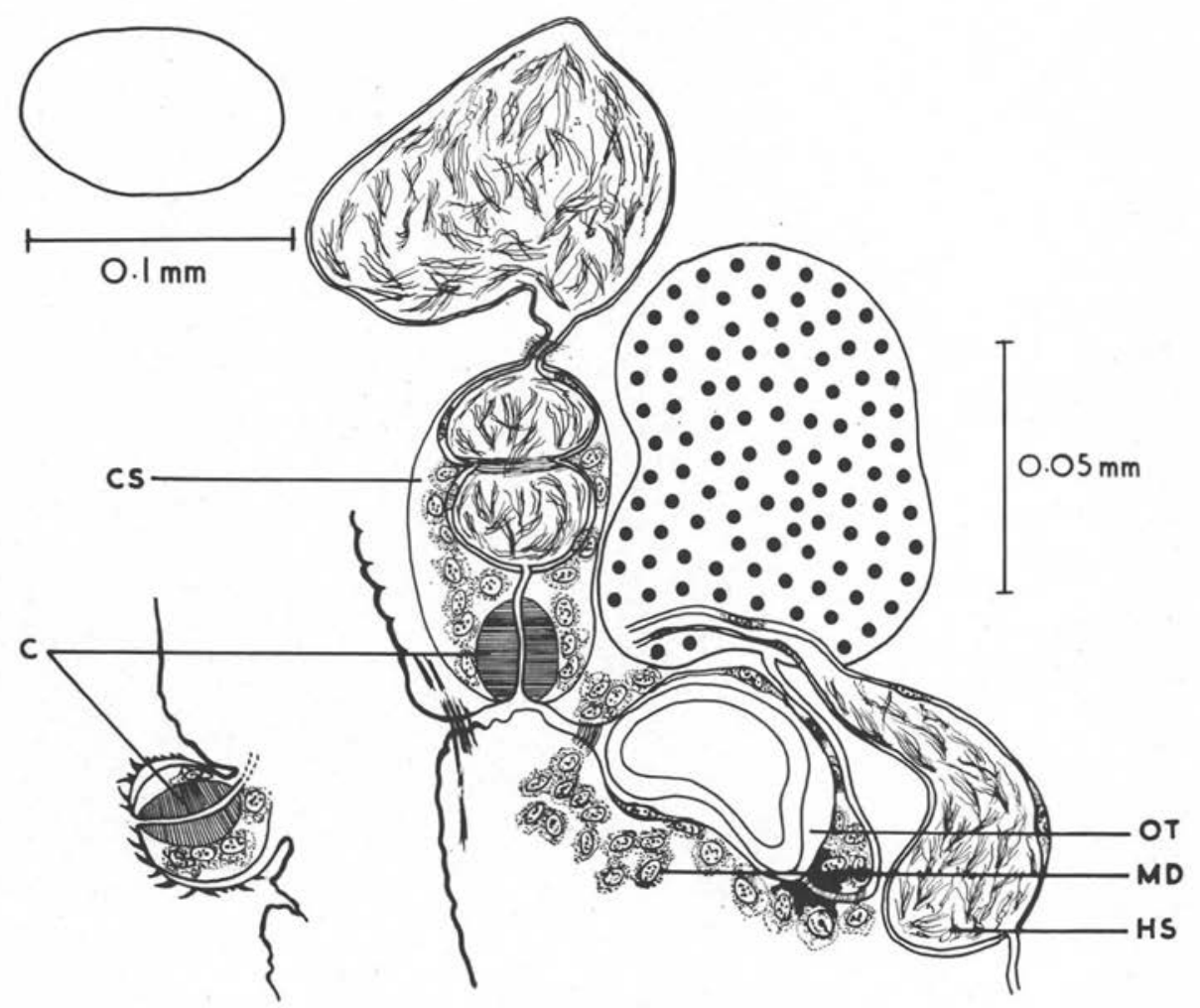

Aвв. 7: Ei von Spirhapalum elongatum.

Aвв. 8: Ootyp mit umliegenden Geschlechtsgängen von Spirhapalum elongatum. CS = Cirrussack, $\mathrm{C}=$ Cirrus, OT $=$ Ootyp, $\mathrm{MD}=$ Mehlissche Drüse, $\mathrm{HS}=$ Hintere Samenblase.

Körperseite, Laurerscher Kanal vorhanden, Uterus besteht nur aus einem ovalen Ootyp, der in einen Metraterm übergeht und sich nahe der männlichen Geschlechtsöffnung nach aussen öffnet. Zahlreiche Dotterfollikel zwischen der Darmverzweigung und dem hinteren Körperende, sowohl zwischen als auch lateral von den Darmschenkeln. Im Gebiet der hinteren Hoden gehen die rechten Dotterstöcke in die linken übr. Parasiten des Blutgefässystems von Süsswasser-Schildkröten.

Die einzige bisher beschriebene Art, S. polesianum Ejsmont, 1927 aus Emys orbicularis in Polen ist in den folgenden Merkmalen von der neuen Art verschieden. $1.18-2.3 \times 0.38-0.65 \mathrm{~mm}$ gross, Mundsaugnapf $0.069-0.076 \times 0.060-0.085 \mathrm{~mm}$, Acetabulum 0.074-0.123 mm Durchmesser. Das Ovar ist grösser als die Teilhoden, die Vesicula seminalis liegt hauptsächlich vor dem Ovar, der Körper ist gedrungener. 


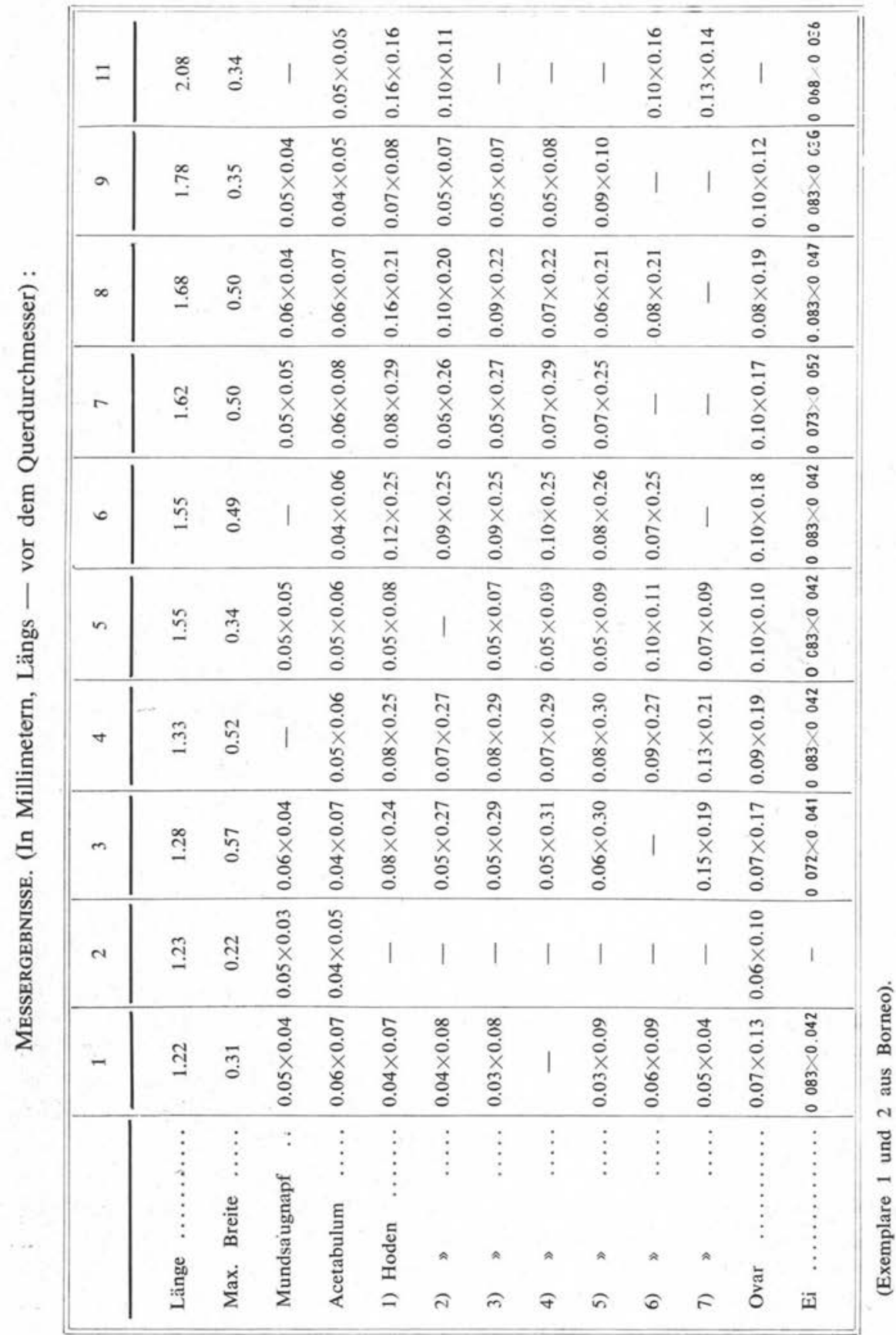




\section{Zusammenfassung.}

Die folgenden malayischen Trematoden werden beschrieben: Zonorchis sp. aus Callosciurus notatus und C. caniceps, Coeuritrema macrotesticularis n. sp. aus Dogania subplana, Spirhapalum elongatum n. sp. aus Cyclemys amboinensis.

\section{Bibliographie}

ZONORCHIS n. sp.

Breues R. R., und Jimenez Quiros 0., 1959. - Helmintos de la Republica de Costa Rica XII. Zonorchis costarricensis n. sp. (Trematoda, Dicrocoeliidae) parasito de vias biliares de Gymnostinops montezuma (Icteridae). Rev. Biol. Trop. Univ. Costa Rica, 7, 125-129.

JAiswal G. P., 1957. - Studies on the Trematode Parasites of fishes and birds found in Hyderabad State. Pt. I-IV. Zool. Jahrb. Abtlg. System., Ökol., Geogr. der Tiere, $85,1-72$.

SkrJabin K. I., 1952. - Die Trematoden der Tiere und des Menschen, Bd. 7, (In Russisch). COEURITREMA MACROTESTICULARIS n. sp.

SkrJabin K. I., 1951. - Die Trematoden der Tiere und des Menschen, Bd. 5, (In Russisch). Yamaguti S., 1958. - Systema helminthum, vol. 1.

SPIRHAPALUM ELONGATUM n. sp.

Skrjabin K. I., 1951. - Die Trematoden der Tiere und des Menschen, Bd. 5, (In Russisch). 